\title{
Sarcoid Myositis with Anti-Ku Antibody Consistent with both Sarcoidosis and Polymyositis
}

\author{
Nobuyasu Awano ${ }^{1}$, Kensuke Fukuda ${ }^{1}$, Masashi Sakayori ${ }^{1}$, Keisuke Kondoh ${ }^{1}$, \\ Ryu Ono ${ }^{1}$, Atsuko Moriya ${ }^{1}$, Tsunehiro Ando ${ }^{1}$, Toshio Kumasaka ${ }^{2}$, \\ Tamiko Takemura ${ }^{2}$ and Soichiro Ikushima ${ }^{1}$
}

\begin{abstract}
We herein describe a case of sarcoid myositis with anti-Ku antibody positivity. Pathological findings of the muscle were compatible with sarcoidosis, but could not be completely distinguished from myositis diseases that arise from other causes. According to a physical examination, pathological findings, the detection of anti$\mathrm{Ku}$ antibody and the human leukocyte antigen (HLA)-DPB1 allele, we strongly suspected that the patient developed both sarcoidosis and polymyositis. Sarcoidosis is often complicated by autoimmune diseases. This case suggests the possibility that sarcoidosis and other autoimmune diseases may have common causal genetic factors.
\end{abstract}

Key words: sarcoidosis, polymyositis, anti-Ku antibody, overlap syndrome, HLA-DPB1

(Intern Med 55: 2049-2053, 2016)

(DOI: 10.2169/internalmedicine.55.5816)

\section{Introduction}

Sarcoidosis is a systemic granulomatous disease of unknown origin. It involves many organs such as the eyes, lungs, lymph nodes, heart, and musculoskeletal system. Patients with acute sarcoid myositis complain of muscle weakness or muscle atrophy. It is difficult to differentiate sarcoid myositis from other myositis diseases that arise from other causes. Sarcoidosis frequently complicates autoimmune diseases, such as rheumatoid arthritis (1), systemic scleroderma (2), and polymyositis/dermatomyositis (PM/ DM) (3-7). PM/DM causes symmetrical muscle weakness and muscle atrophy. Although PM/DM shows no granuloma in the muscle, its clinical and pathological features are similar to sarcoid myositis.

The presence of anti-Ku antibody was initially reported in 1981 by Mimori et al. in systemic sclerosis-dermatomyositis overlap syndrome (8). Subsequently, anti-Ku antibodies have been detected in various connective tissue diseases including systemic lupus erythematosus, Sjögren's syndrome and mixed connective tissue diseases (MCTD) $(9,10)$. We herein report a rare case of sarcoid myositis with anti-Ku antibody positivity that strongly suggests the occurrence of both sarcoidosis and polymyositis.

\section{Case Report}

A 30-year-old man was referred to our hospital in 2011. The patient was diagnosed with sarcoidosis in a previous hospital in 2006. The first part of the patient's clinical course has been previously reported (11) and will be briefly described here. His initial symptoms were progressive proximal extremity weakness, general fatigue, mild exertional dyspnea and poor vision. The body weight of the patient was $60 \mathrm{~kg}$ and he did not lose weight. The patient did not develop arthralgia, Raynaud's phenomenon or skin disease except for eczema on the back. The presentation of uveitis, bilateral hilar and mediastinal lymphadenopathy on chest Xray and a CT scan, a negative tuberculin skin test and elevated levels of serum angiotensin-converting enzyme concentration (ACE) (28.3 U/L), lymphocytes (36\%) and CD4/

${ }^{1}$ Department of Respiratory Medicine, Japanese Red Cross Medical Center, Japan and ${ }^{2}$ Department of Pathology, Japanese Red Cross Medical Center, Japan

Received for publication May 22, 2015; Accepted for publication November 10, 2015

Correspondence to Dr. Nobuyasu Awano, awanobu0606@hotmail.co.jp 


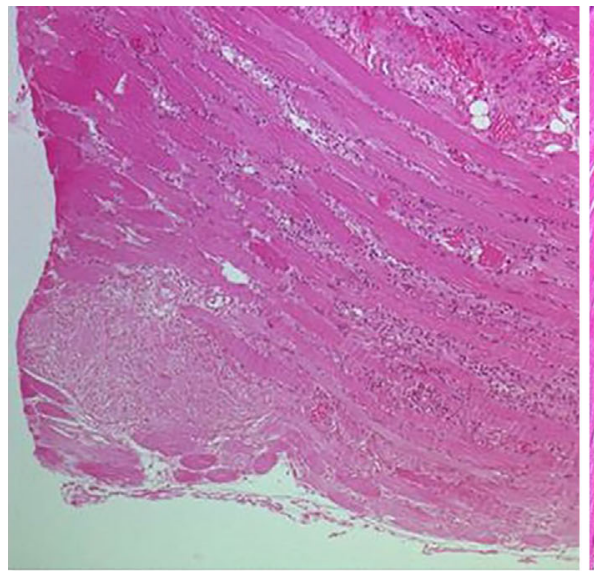

A

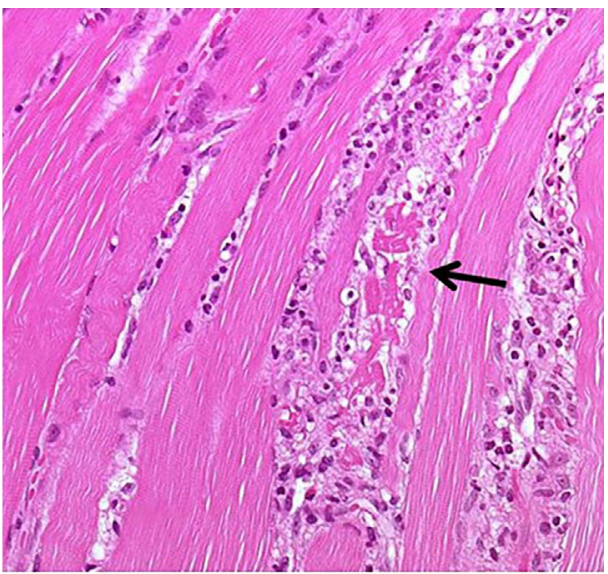

B

Figure 1. A) Pathological findings of specimens obtained from the quadriceps muscle revealed noncaseating epithelioid cell granulomas and endomysial mononuclear cell infiltration [Hematoxylin and Eosin (H\&E) staining, 20×]. B) Endomysial infiltration of mononuclear cells, different sizes of muscle fiber and muscle necrosis (arrow) were detected (H\&E staining, 200x).

CD8 ratio (4.1) in the bronchoalveolar lavage fluid were compatible with sarcoidosis. Transbronchial lung biopsies, skin biopsies and a specimen of the quadriceps showed multiple non-caseating epithelioid cell granulomas. According to these findings, the patient was diagnosed with sarcoidosis involving the parotid and lachrymal glands, eyes, lungs, lymph nodes and muscles. A physical examination showed symmetrical proximal muscle weakness and atrophy and the serum levels of creatine kinase (CK) and aldolase were elevated to $9,838 \mathrm{IU} / \mathrm{L}$ and $243 \mathrm{IU} / \mathrm{L}$, respectively. According to the myopathic pattern of electromyography, a diffuse high-signal intensity area on short T1-weighted inversionrecovery images in magnetic resonance imaging of the bilateral thighs and pathological findings of the quadriceps (Fig. 1), the patient was diagnosed with sarcoid myositis. Both prednisolone (PSL) and steroid pulse therapy (methylprednisolone sodium $1,000 \mathrm{mg} / \mathrm{d}$ for three days) were ineffective. The addition of methotrexate (MTX) $(7.5 \mathrm{mg}$ once a week) was effective. The serum levels of CK and aldolase decreased to within the normal levels and the patient's muscle weakness improved. The details of this clinical course have been previously reported (11). In January 2011, the patient moved and visited our hospital.

At the first visit, the patient had been taking $7 \mathrm{mg}$ /day PSL and $4 \mathrm{mg}$ /week MTX but complained of proximal extremity weakness. The manual muscle testing score was $4 / 5$ for both the upper and lower extremities. Uveitis was wellcontrolled by eye drops, while bilateral hilar and mediastinal lymphadenopathy remained present on CT. The laboratory data showed an ACE level of 18.0 IU/L that was within the normal limits, however, the $\mathrm{CK}$ and aldolase levels had increased to 2,131 U/L and 33.9 IU/L, respectively. Although the patient did not show Raynaud's phenomenon, scleroderma, hyperpigmentation or joint diseases, antinuclear antibody (ANA) testing was positive at a titer of 1280 (speckled type). Therefore, we decided to further analyze his autoimmune antibodies. Subsequently, anti-Ku antibody was detected by a radioimmunoprecipitation assay, but other myositis-specific, myositis-associated antibodies, including anti-Jo-1 antibody, were all negative (Table). Furthermore, the human leukocyte antigen (HLA)-DPB1 allele was identified as *05:01,*09:01:01. Muscle disease manifestation, the elevation of the serum $\mathrm{CK}$, aldolase and C-reactive protein (CRP) levels, the myopathic pattern of electromyography and the pathological findings of the quadriceps in 2006 (Fig. 1,2) were compatible with polymyositis. According to these findings, we speculated that the patient had developed both sarcoidosis and polymyositis.

The same dosages of PSL and MTX were continued, however, the patient's muscle weakness gradually worsened. As the serum level of $\mathrm{CK}$ had increased to 7,242 U/L in January 2012, the dosages of PSL and MTX were increased to $40 \mathrm{mg} /$ day and $6 \mathrm{mg} /$ week, respectively. The serum CK level subsequently improved, but muscular disease manifestation could not be completely controlled. In order to reduce the side effects of PSL, the dosage of PSL was gradually decreased, while the dosage of MTX was gradually increased to $14 \mathrm{mg} /$ week. Taking PSL $7 \mathrm{mg}$ /day and MTX 14 $\mathrm{mg} /$ week, the serum levels of CK and aldolase decreased to within the normal levels and the patient's condition is presently stable as of February 2015 (Fig. 3).

\section{Discussion}

Sarcoidosis can affect any organ, but symptomatic musculoskeletal involvement is rare (12). Muscle involvement is classified into three types: chronic myopathy, palpable nodular type and acute sarcoid myositis (13). The present patient was diagnosed with sarcoid myositis due to acute proximal muscle weakness and pathological findings, such as non- 
Table. Laboratory Data in January 2011.

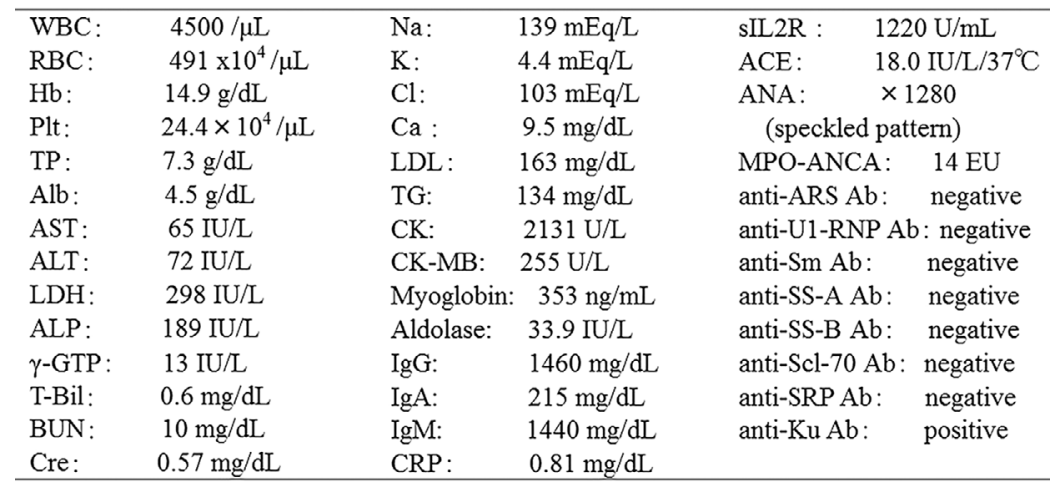

Reference ranges: myoglobin: 18-70ng/mL, aldolase: 1.7-5.7IU/L, ACE: 8.3-21.4IU/L, MPO-ANCA: 0-19EU.

ANA: antinuclear antibody, sIL2R: soluble IL-2 receptor, Ab: antibody, ARS: aminoacyltRNA synthetase

Measurement methods: anti-Jo-1 Ab, anti-ARS Ab, anti-SRP Ab and anti-Ku Ab: radioimmunoprecipitation assays.

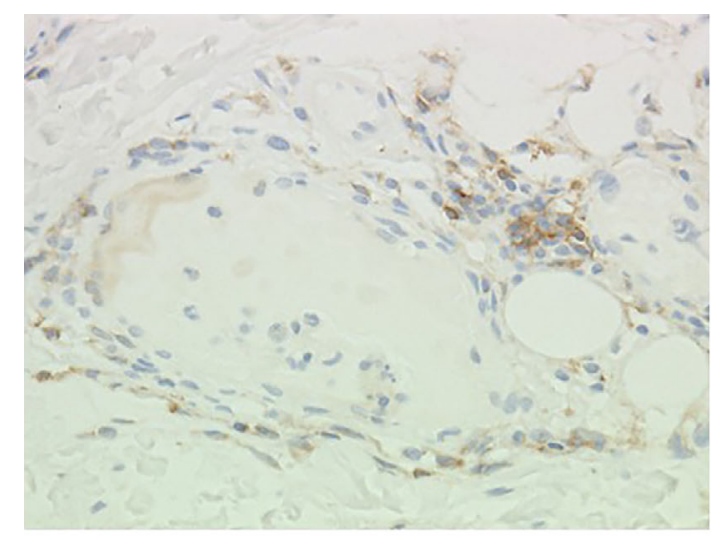

A

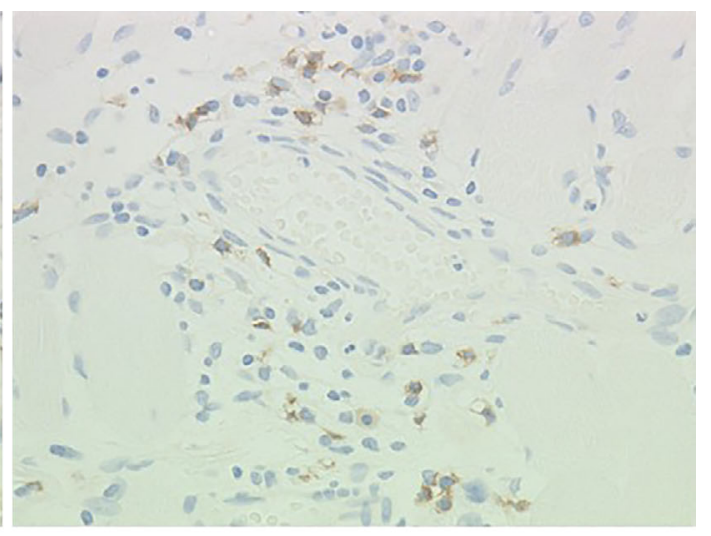

B

Figure 2. Both CD4 positive $\mathrm{T}$ cell $(\mathrm{A})$ and $\mathrm{CD8}$ positive $\mathrm{T}$ cell $(\mathrm{B})$ infiltration into the muscles were detected (immunohistochemical staining, 40x). The CD4/CD8 ratio was 0.5 .

caseating epithelioid cell granulomas and lymphocytic inflammation.

Furthermore, according to the criteria proposed by Bohan and Peter in 1975 (14), the patient's findings were all compatible with polymyositis. The pathological findings revealed not only non-caseating epithelioid cell granulomas, but also diffuse lymphocyte infiltration, muscle necrosis and myolysis. We considered that these pathological changes were caused by both sarcoid myositis and polymyositis.

In addition, we detected not only ANA, but also anti-Ku antibody. $\mathrm{Ku}$ antigen is a heterodimer composed of $70 \mathrm{kD}$ and $80 \mathrm{kD}$ subunits that bind to the ends of double-stranded DNA through its zipper structure (15). It plays a role in the repair of damaged DNA $(16,17)$ and $\mathrm{V}(\mathrm{D}) \mathrm{J}$ recombination in $\mathrm{B}$ and $\mathrm{T}$ cell activation (18).

In the last several years, a further genotypic analysis of patients with anti-Ku antibodies has been performed. Hirakata et al. examined the HLA class II (DRB1, DQA1, DQB1 and DPB1) of 21 Japanese patients with anti-Ku antibody-positive connective tissue diseases. They showed that HLA-DPB $1 * 05: 01$ was detected in all 21 patients and that the allele was one of the most common allele types in Japanese patients with anti-Ku antibody (19). However, HLA-DPB $1 * 05: 01$ was detected in 59\% of the control subjects. Therefore, it does not necessarily serve as a useful diagnostic tool. Another study found that HLA-DPB1 *05:01 was also a risk factor for Graves' disease in Japan (20). Hirakata et al. suggested that there is a common immunogenetic background for Graves' disease and the anti-Ku autoimmune response. In the present case, the HLA-DPB1 allele was identified as *05:01, *09:01:01. Several studies reported a weak association between HLA alleles and sarcoidosis. Glutamic acid at position 69 in HLA-DPB1 was reported to be associated with sarcoidosis (21). HLA-DPB1*05:01 does not have glutamic acid at position 69, however, HLA-DPB1 *09:01 does. On the other hand, another study revealed a strong association between glutamic acid at position 69 in HLA-DPB1 and chronic beryllium disease (22). It has been 


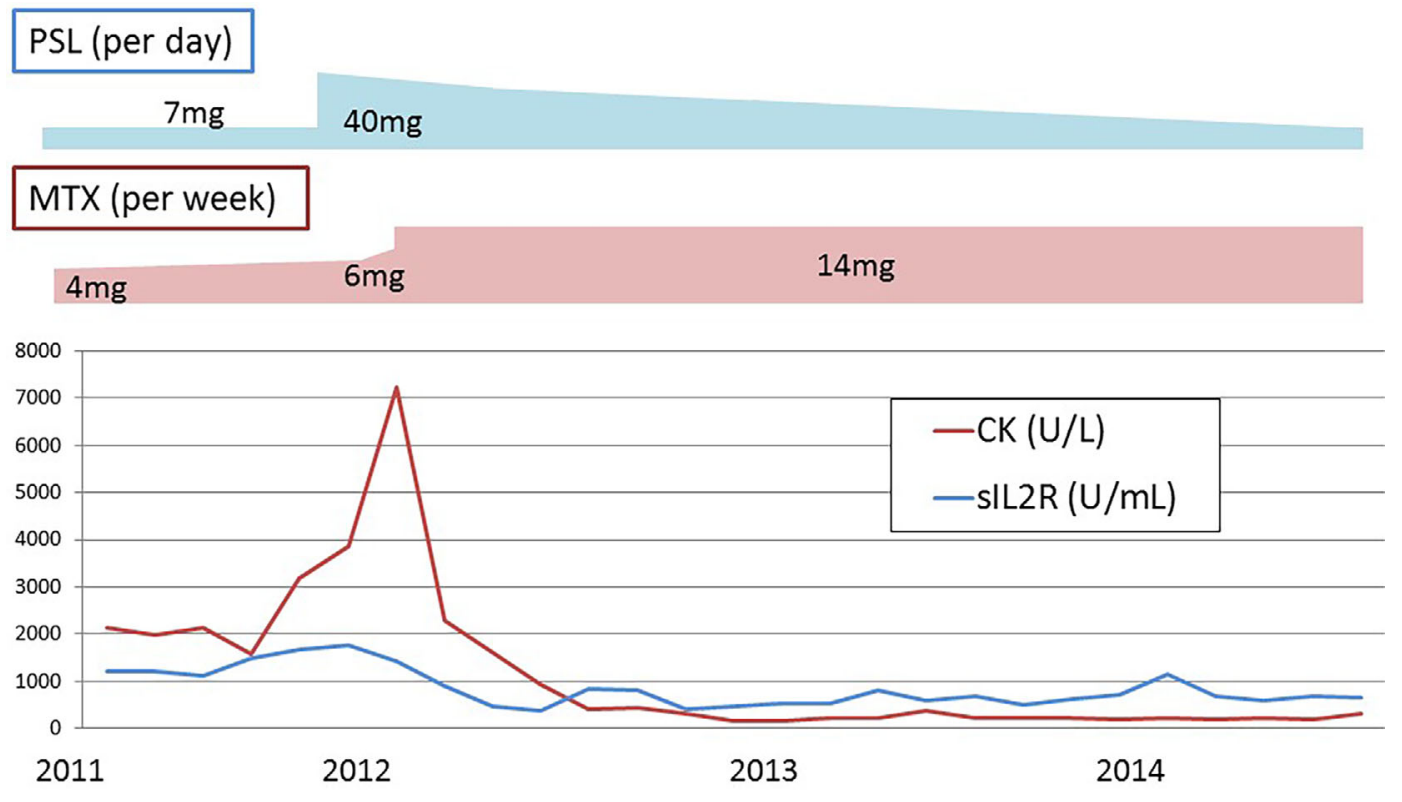

Figure 3. The patient's clinical course. PSL: prednisolone, MTX: methotrexate, sIL2R: soluble IL-2 receptor

suggested that HLA-DPB1 may play an important role in antigen presentation and recognition in chronic granulomatous diseases. Similarly, our findings suggest that the immunogenetic background may affect the occurrence of both sarcoidosis and polymyositis. Sarcoidosis infrequently complicates PM/DM, however, we have found only one case of sarcoidosis that is anti-Ku antibody-positive and accompanied by polymyositis (3). The patient developed systemic sclerosis, polymyositis, autoimmune hepatitis and sarcoidosis, which suggests that these autoimmune diseases have a similar immunopathogenic mechanism. The etiology of sarcoidosis is currently unknown. Genetic susceptibility to environmental agents and endogenous infection of pathogens such as Propionibacterium acnes may be involved in the etiology of sarcoidosis (23). Further studies are needed to define the etiology and mechanisms of sarcoidosis.

Corticosteroid therapy is usually beneficial for both sarcoid myositis and inflammatory myopathies of anti-Ku antibody-positive patients (24). However, PSL and steroid pulse therapy were ineffective in the present case. The additional use of MTX showed significantly improved treatment efficacy. A previous study by Chinoy et al. examined the risk of cancer-associated myositis (25). They showed that the detection of anti-Ku antibody in myositis is associated with a lower frequency of cancer as well as a favorable prognosis. In the present case, however, a long-term followup is essential due to the risk of developing malignant lymphoma associated with sarcoidosis or MTX, as well as cancer-associated myositis.

The authors state that they have no Conflict of Interest (COI).

\section{Acknowledgement}

We would like to thank Shota Nakajima, MD (Second Depart- ment of Internal Medicine, University of Nagasaki) for valuable support while carrying out this study.

\section{References}

1. Josse $S$, Klemmer N, Moreno-Swirc S, et al. Infliximab induced skin and pulmonary sarcoidosis in a rheumatoid arthritis patient. Joint Bone Spine 76: 718-719, 2009.

2. Ren XX, Hou SW, Li ZF, et al. A case of sarcoidosis with systemic sclerosis. Chin Med J (Engl) 126: 4400, 2013.

3. Lis-Swiety A, Brzezinska-Wcislo L, Pierzchala E, et al. Systemic sclerosis-polymyositis overlap syndrome accompanied by autoimmune hepatitis and sarcoidosis of mediastinal lymphnodes. J Eur Acad Dermatol Venereol 20: 107-108, 2006.

4. Hart Y, Schwartz MS, Bruckner F, et al. Relapsing dermatomyositis associated with sarcoidosis. J Neurol Neurosurg Psychiatry 51: 311-313, 1988.

5. Nishioka M, Igawa K, Yahata Y, et al. Simultaneous occurrence of dermatomyositis and systemic sarcoidosis with recurrent breast cancer. J Dermatol 39: 485-486, 2012.

6. Hayashi T, Nakamura T, Kurachi K, et al. Ulcerative colitis accompanied with sarcoidosis and dermatomyositis: report of a case. Dis Colon Rectum 51: 474-476, 2008.

7. Brateanu AC, Caracioni A, Smith HR. Sarcoidosis and dermatomyositis in a patient with hemoglobin SC. A case report and literature review. Sarcoidosis Vasc Diffuse Lung Dis 17: 190-193, 2000.

8. Mimori T, Akizuki M, Yamagata $\mathrm{H}$, et al. Characterization of a high molecular weight acidic nuclear protein recognized by autoantibodies in sera from patients with polymyositis-scleroderma overlap. J Clin Invest 68: 611-620, 1981.

9. Cavazzana I, Ceribelli A, Quinzanini M, et al. Prevalence and clinical associations of anti-Ku antibodies in systemic autoimmune diseases. Lupus 17: 727-732, 2008.

10. Franceschini F, Cavazzana I, Generali D, et al. Anti-Ku antibodies in connective tissue diseases: clinical and serological evaluation of 14 patients. J Rheumatol 29: 1393-1397, 2002.

11. Fujita H, Ishimatsu $Y$, Motomura $M$, et al. A case of acute sarcoid myositis treated with weekly low-dose methotrexate. Muscle Nerve 44: 994-999, 2011. 
12. Mayock RL, Bertrand P, Morrison CE, Scott JH. Manifestations of sarcoidosis. analysis of 145 patients, with a review of nine series selected from the literature. Am J Med 35: 67-89, 1963.

13. Stjernberg N, Cajander S, Truedsson H, et al. Muscle involvement in sarcoidosis. Acta Med Scand 209: 213-216, 1981.

14. Bohan A, Peter JB. Polymyositis and dermatomyositis (first of two parts). N Engl J Med 292: 344-347, 1975.

15. Mimori T, Ohosone $\mathrm{Y}$, Hama $\mathrm{N}$, et al. Isolation and characterization of cDNA encoding the $80-\mathrm{kDa}$ subunit protein of the human autoantigen $\mathrm{Ku}$ (p70/p80) recognized by autoantibodies from patients with scleroderma-polymyositis overlap syndrome. Proc Natl Acad Sci U S A 87: 1777-1781, 1990.

16. Mimori T, Hardin JA, Steitz JA. Characterization of the DNAbinding protein antigen $\mathrm{Ku}$ recognized by autoantibodies from patients with rheumatic disorders. J Biol Chem 261: 2274-2278, 1986.

17. Mimori T, Hardin JA. Mechanism of interaction between $\mathrm{Ku}$ protein and DNA. J Biol Chem 261: 10375-10379, 1986.

18. Smider V, Rathmell WK, Lieber MR, Chu G. Restoration of X-ray resistance and $\mathrm{V}(\mathrm{D}) \mathrm{J}$ recombination in mutant cells by $\mathrm{Ku}$ cDNA Science 266: 288-291, 1994.
19. Hirakata M, Suwa A, Kuwana M, et al. Association between autoantibodies to the $\mathrm{Ku}$ protein and DPB1*. Arthritis Rheum 52: 668-669, 2005.

20. Dong RP, Kimura A, Okubo R, et al. HLA-A and DPB1 loci confer susceptibility to Graves' disease. Hum Immunol 35: 165-172, 1992.

21. Lypmany PA, Petrek M, Southcott AM, et al. HLA-DPB polymorphisms: Glu 69 association with sarcoidosis. Eur J Immunogenet 23: 353-359, 1996.

22. Richeldi L, Sorrentino R, Saltini C. HLA-DPB1 glutamate 69: a genetic marker of beryllium disease. Science 262: 242-244, 1993.

23. Eishi Y, Suga M, Ishige I, et al. Quantitative analysis of mycobacterial and propionibacterial DNA in lymph nodes of Japanese and European patients with sarcoidosis. J Clin Microbiol 40: 198-204, 2002.

24. Rigolet A, Musset L, Dubourg O, et al. Inflammatory myopathies with anti-Ku antibodies: a prognosis dependent on associated lung disease. Medicine (Baltimore) 91: 95-102, 2012.

25. Chinoy $\mathrm{H}$, Fertig $\mathrm{N}$, Oddis $\mathrm{CV}$, et al. The diagnostic utility of myositis autoantibody testing for predicting the risk of cancerassociated myositis. Ann Rheum Dis 66: 1345-1349, 2007.

(C) 2016 The Japanese Society of Internal Medicine http://www.naika.or.jp/imonline/index.html 